\title{
Investigation of parent-of-origin effects induced by fenofibrate treatment on triglycerides levels
}

\author{
Chloé Sarnowski*, Samantha Lent and Josée Dupuis \\ From Genetic Analysis Workshop 20 \\ San Diego, CA, USA. 4-8 March 2017
}

\begin{abstract}
Background: Genome-wide association studies performed on triglycerides (TGs) have not accounted for epigenetic mechanisms that may partially explain trait heritability.

Results: Parent-of-origin (POO) effect association analyses using an agnostic approach or a candidate approach were performed for pretreatment TG levels, posttreatment TG levels, and pre- and posttreatment TG-level differences in the real GAW20 family data set. We detected 22 genetic variants with suggestive POO effects with at least 1 phenotype $\left(P \leq 10^{-5}\right)$. We evaluated the association of these 22 significant genetic variants showing POO effects with close DNA methylation probes associated with TGs. A total of 18 DNA methylation probes located in the vicinity of the 22 SNPs were associated with at least 1 phenotype and 6 SNP-probe pairs were associated with DNA methylation probes at the nominal level of $P<0.05$, among which 1 pair presented evidence of POO effect. Our analyses identified a paternal effect of SNP rs301621 on the difference between pre- and posttreatment TG levels $\left(P=1.2 \times 10^{-5}\right)$. This same SNP showed evidence for a maternal effect on methylation levels of a nearby probe (cg10206250; $P=0.01$ ). Using a causal inference test we established that the observed POO effect of rs301621 was not mediated by DNA methylation at cg10206250.

Conclusions: We performed POO effect association analyses of SNPs with TGs, as well as association analyses of SNPs with DNA methylation probes. These analyses, which were followed by a causal inference test, established that the paternal effect at the SNP rs301621 is induced by treatment and is not mediated by methylation level at cg10206250.
\end{abstract}

Keywords: Triglycerides, Fenofibrate treatment, Epigenetics, Parent-of-origin effects, DNA methylation

\section{Background}

Despite the success of genome-wide association studies (GWAS) conducted for lipid levels, particularly triglyceride (TG) levels, to discover common risk alleles, the genetic factors identified to date only account for a small part of the genetic variance of this trait. Genetic studies have largely focused on DNA sequence variations. Sources of this unexplained heritability might include epigenetic mechanisms such as DNA methylation. Epigenetic modifications are heritable changes influencing

* Correspondence: chloesar@bu.edu

Department of Biostatistics, Boston University School of Public Health, 801 Massachusetts Avenue, Boston, MA 02118, USA gene expression without alterations of the underlying DNA sequence. Few epigenome-wide studies have been conducted for lipid levels, particularly TG levels [1-6]. However, DNA methylation can cause partial or complete silencing of 1 parental allele at a specific locus and can lead to a differential effect of that polymorphism on disease in offspring according to the parental origin of the risk allele (parent-of-origin [POO] effect). Such epigenetic mechanism has been described for lipid levels by Predazzi et al., who reported sex-specific parental effects on offspring lipid levels [7].

Our approach consisted in combining POO effect associations with association of nearby DNA methylation 
probes with TGs followed by a causal inference test to assess whether methylation mediates the observed POO. This approach has been successful in identifying differentially methylated cytosine-phosphate-guanine (CpG) sites that mediate the association between a genetic variant and a phenotype under a POO effect [8].

We performed $\mathrm{POO}$ effect association analyses of pretreatment TG levels, posttreatment TG levels, and preand posttreatment TG-level differences in the real GAW20 family data set, using a linear regression approach implemented in the quantitative transmission disequilibrium test (QTDT) software [9]. We conducted 2 approaches to select suggestive POO effect associations: a candidate approach in regions known to be associated with TG levels, and an agnostic approach over the full genome. We then investigated whether the detected POO effects were associated with TG-associated DNA methylation probes and performed causal inference tests. Figure 1 shows the analytical strategy and the biological mechanism of mediation by DNA methylation of the association between the genetic variant and the phenotype (TG).

\section{Methods}

\section{Triglyceride phenotype transformations}

The real data example for GAW20 is from the Genetics of Lipid Lowering Drugs and Diet Network (GOLDN) study data set [4]. This study ascertained and recruited families from the Family Heart Study at 2 centers, Minneapolis, MN and Salt Lake City, UT, who self-reported to be white. TG levels in the GAW20 data set were measured at 4 exams: 2 pretreatment exams (visits 1 and 2) and 2 posttreatment exams (visits 3 and 4). At visit 1 , subjects were measured after an overnight fast with a standard lipid profile. The next day, they returned to clinic, again fasting, for a second, repeat lipid profile. All subjects were then given the fenofibrate drug for a 3-week treatment period, after which they returned to the clinic for 2 consecutive days of lipid profiling (visits 3 and 4, both with overnight fasting), to assess the response to treatment. We performed association analyses of single-nucleotide polymorphisms (SNPs) with the average natural-log TG pretreatment $\left(\mathrm{TG}_{1-2}\right)$, the average natural-log $\mathrm{TG}$ posttreatment $\left(\mathrm{TG}_{3-4}\right)$, and the natural-log TG difference between pre- and posttreatment $\left(\Delta \mathrm{TG}_{1-4}\right)$ using a linear mixed-effect model to account for familial correlations and adjusted for age, sex, center $(0=$ Minnesota, $1=\mathrm{Utah})$, and smoking (coded as never, former, or current).

\section{Association analysis of SNPs with TG level}

GWAS genotyping was performed on DNA extracted from blood drawn at baseline, using the Affymetrix Genome-wide Human SNP Array 6.0. Association analyses were conducted using the QTDT software with and without accounting for POO effects. From the real data, we analyzed 823 genotyped individuals from 173 families (715,787 SNPs). We evaluated the association of principal components calculated in the GAW20 data set with the traits evaluated in this study. As population stratification was not expected to be a major concern, we used the QTDT -at option to perform a total association analysis [9]. This model is not a transmission disequilibrium test and has a considerably higher power and greater efficiency to detect POO effects. The trait mean of child $j$ in family $i$ is modeled as: $Y_{i j}=\alpha+\beta_{G} G_{i j}+e_{i j}$ where $\beta_{G}$ is the genetic effect of the minor allele $(a)$ of the SNP and $G_{i j}$ is the number of minor alleles carried by child $j$ in family $i$.

Then, our association analyses focused on POO effects on TG in the offspring. This approach involves modeling the phenotype as a function of covariates and genotypes, taking parental original of each allele into consideration [10]. We use the QTDT with POO effect options (-of, $-o t,-o m$, and $-o p$ ) to get estimates of parental origin of the alleles [9]. The model for the mean of the trait of child $j$ in family $i$ is: $Y_{i j}=\alpha+\beta_{\text {mat }} G_{1 i j}+\beta_{p a t} G_{2 i j}+e_{i j}$ where $\beta_{\text {mat }}$ is the genetic effect of a maternally inherited minor allele, $\beta_{\text {pat }}$ is the genetic effect of a paternally minor allele, $G_{1 i j}$ is the estimated number of minor allele
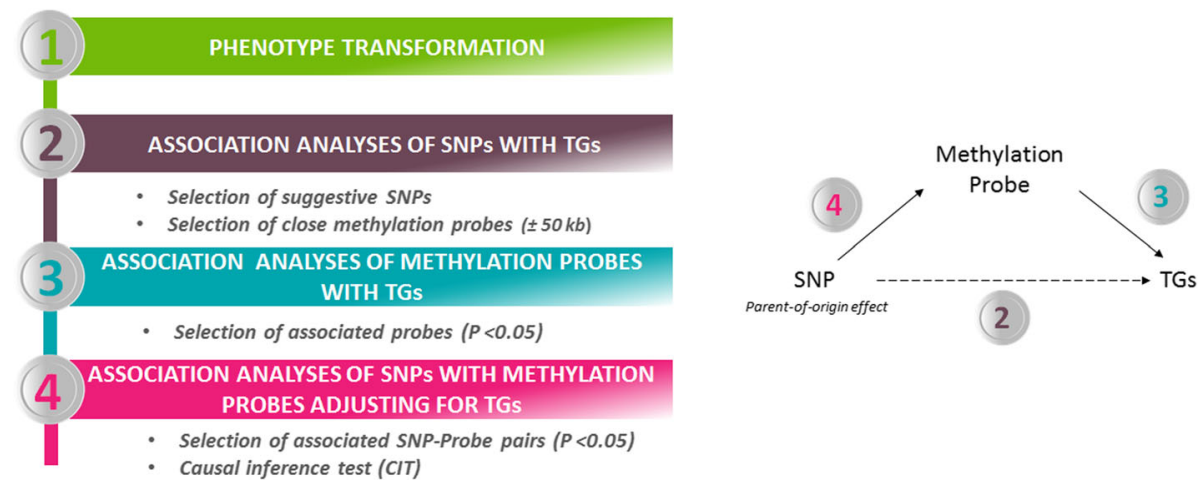
inherited from the mother, and $G_{2 i j}$ is the estimated number of minor alleles inherited from the father. The -at -ot tests for difference between paternal and maternal transmissions $\left(\beta_{\text {pat }}=\beta_{\text {mat }}\right)$, the $-a t$-of uses a saturated model where maternal and paternal inherited alleles are modeled separately $\left(\beta_{\text {pat }}=\beta_{\text {mat }}=0\right)$, whereas the $-a t-o m$ and $-a t-o p$ tests for maternal effect $\left(\beta_{\text {mat }}=\right.$ $0)$ or paternal effect $\left(\beta_{\text {pat }}=0\right)$ only. We modified the QTDT to test 1 parental effect while adjusting for the other parent contribution (om_op and op_om models).

Selection of suggestive POO effects was performed using 2 different approaches. We first used a candidate approach in regions known to be associated with TG levels using the GWAS catalog (http://www.ebi.ac.uk/gwas/search?query=triglycerides). We selected 14 European-ancestry GWAS after excluding GWAS performed on specific phenotypes (responses to treatment) or related phenotypes (obesity, Type 2 diabetes, lipids). A total of 129 variants in 59 regions were reported associated with TG levels at $P<10^{-5}$ (threshold to include associations in the GWAS catalog). We used $\mathrm{a} \pm 50-\mathrm{kb}$ window around the reported SNPs to define candidate loci. We used an arbitrary threshold of $10^{-5}$ to declare a SNP association suggestive under the POO effect model (in at least one of the following models: ot, of, om, op, om_op, op_om). We then used an agnostic approach over the full genome with the same arbitrary threshold of $10^{-5}$ to declare a SNP association suggestive under the POO effect model (in at least 1 of the following models: ot, of, om, op, om_op, op_om).

Association analyses of DNA methylation probes with TGs DNA was extracted from CD4+ T cells and the proportion of sample methylation at $>450,000 \mathrm{CpG}$ sites was quantified by using the Illumina Infinium Human Methylation $450 \mathrm{~K}$ BeadChip from blood drawn in both visit 2 and visit 4 for each participant. We selected DNA methylation probes located within $\mathrm{a} \pm 50-\mathrm{kb}$ window around the suggestive SNPs under the POO effect model. We performed epigenetic association analyses of natural-log $\mathrm{TG}$ pretreatment $\left(\mathrm{TG}_{2}\right)$, natural-log TG posttreatment $\left(\mathrm{TG}_{4}\right)$, and natural-log pre- and posttreatment TG difference $\left(\Delta \mathrm{TG}_{2-4}\right)$ using a linear mixed-effect model adjusted for age, sex, center, and smoking to account for familial correlations. From the real data, we analyzed 679 and 403 individuals from 163 and 136 families with genotypes and methylation available at exam 2 or 4 , respectively. We selected all DNA methylation probes associated with at least 1 trait at the nominal level of $P<0.05$.

\section{Association analyses of SNPs with methylation probes}

Association analysis between DNA methylation probes and SNPs was conducted using the same approach described for SNP-TG association, using the QTDT software to evaluate the association between suggestive SNPs under the POO effect model and significant DNA methylation probes, while including the phenotype in the model.

\section{Causal inference test}

We performed a causal inference test [11] to determine if the detected POO effects were mediated by DNA methylation at nearby probes associated with the phenotypes. The causal inference test comprises 4 conditions that have to be met to conclude that mediation is responsible for the observed association: the SNP and the phenotype are associated; the SNP is associated with methylation after adjusting for the phenotype; the methylation is associated with the phenotype after adjusting for the SNP; and the SNP is independent of the phenotype after adjusting for the methylation [11].

\section{Results}

Using an agnostic approach on the genome, we identified 19 SNPs with a suggestive POO effect $\left(P<10^{-5}\right)$, among which 6 were associated with $\mathrm{TG}_{1-2}, 1$ with $\mathrm{TG}_{3-}$ 4 , and 12 with $\Delta \mathrm{TG}_{1-4}$. Among the SNPs previously reported to be associated with TG level, only 8 were associated with the phenotypes $\left(\mathrm{TG}_{1-2}\right)$ at $P \leq 10^{-3}$ and no SNP showed strong POO effects $\left(P>10^{-3}\right)$. Using a candidate approach, we identified 2 SNPs with a suggestive POO effect $\left(P<10^{-5}\right)$, among which 2 were associated with $\mathrm{TG}_{1-2} \quad$ (rs7559813, $P$ op_om $=8.3 \times 10^{-6}$ and rs10417097, $P$ op_om $=8.0 \times 10^{-6}$ ) and 1 was also associated with $\mathrm{TG}_{3-4}\left(\mathrm{rs} 10417097\right.$, P op_om $\left.=8.5 \times 10^{-7}\right)$. We identified 18 DNA methylation probes located in the vicinity of these SNPs that were associated with the phenotypes at $P<0.05$ (11 with $\mathrm{TG}_{2}, 3$ with $\mathrm{TG}_{4}$, and 4 with $\left.\Delta \mathrm{TG}_{2-4}\right)$. We detected $6 \mathrm{SNP}$-probe pairs with association $(\mathrm{P}<0.05)$ in at least 1 model with QTDT (Table 1 ), among which 1 pair (rs301621-cg10206250) presented evidence of $\mathrm{POO}$ effect. We identified a paternal effect of rs301621 on chromosome 16 on pre- and posttreatment TG difference, $\Delta \mathrm{TG}_{1-4}\left(P\right.$ op_om $\left.=1.2 \times 10^{-5}\right)$ and detected a maternal effect of the same SNP on methylation levels of a nearby probe (cg10206250, P om_op = 0.01). The observed paternal effect of rs301621 remains significant when adjusting for DNA methylation at cg10206250 ( $P$ op_om $\left.=5.5 \times 10^{-5}\right)$ and is also associated with posttreatment TG $(P$ op_om $=0.001)$.

\section{Discussion}

We detected 3 SNPs with POO effect associations that reached the multiple-testing correcting threshold using the agnostic approach $\left(P \leq 5 \times 10^{-8}\right)$ or the candidate approach threshold $\left(P \leq 1.2 \times 10^{-5}\right)$. The tested methylation probes were only associated with the tested 


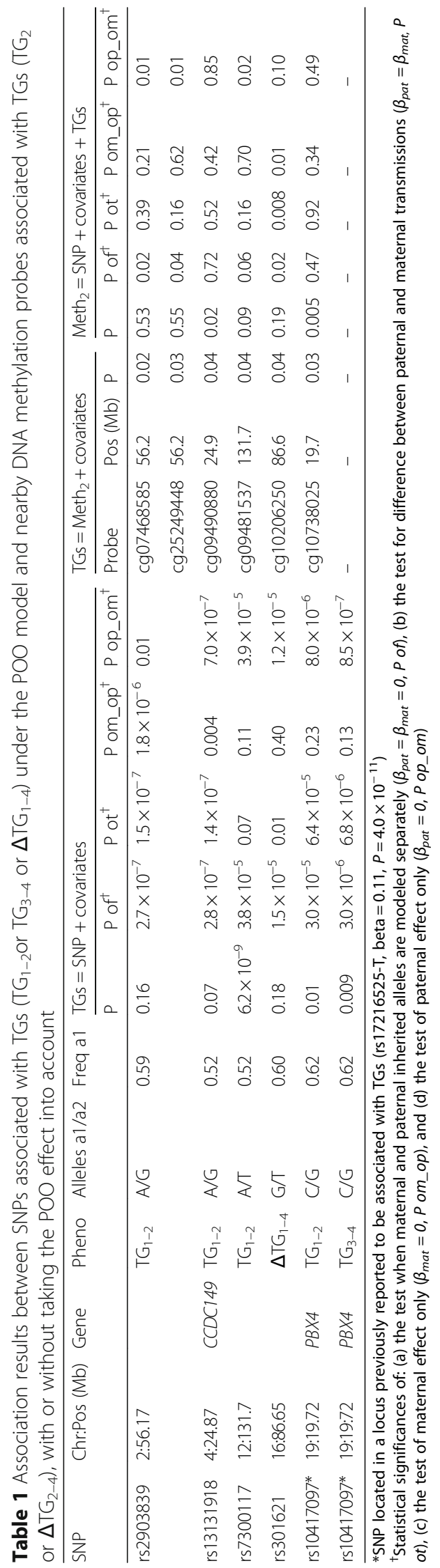


phenotypes before multiple testing correction. This could be from a lack of power because of the small number of individuals analyzed. However, we detected 19 SNPs with suggestive POO effects $\left(P<10^{-5}\right)$ and this may reflect new mechanisms of action of these SNPs, particularly in previously reported loci such as SNP rs10417097 on chromosome 19 in the PBX4 gene [12, 13] with a strong paternal effect on both $\mathrm{TG}_{1-2}$ and $\mathrm{TG}_{3-4}\left(P \leq 10^{-5}\right)$ and associated with methylation levels of cg10738025 ( $P=0.005$, see Table 1$)$. Only one-third of the SNPs previously reported to be associated with TGs were available in the GAW20 data. These SNPs did not present strong POO effects $\left(P>10^{-3}\right)$ and few of them were strongly associated with the phenotypes at $\mathrm{P} \leq 10^{-}$ ${ }^{3}$. This could be from a lack of power because of the small number of individuals analyzed.

\section{Conclusions}

We performed association analyses in the real GAW20 data set to identify POO effects on TG levels in offspring. These analyses were followed by association analyses of SNPs with DNA methylation probes adjusting for TG levels. Interestingly, the G allele of rs301621 was associated with pre- and posttreatment TG difference when transmitted by the father and with methylation levels of cg10206250 when transmitted by the mother. We showed that this paternal effect is induced by treatment. Using a causal inference test, we demonstrated that the observed POO effect of rs301621 on the difference between pre- and posttreatment TG level was not mediated by DNA methylation at cg10206250. Further investigation in a larger number of participants is needed to confirm the POO effects identified in this report.

\section{Abbreviations}

CpG: Cytosine-phosphate-guanine; GOLDN: Genetics of lipid lowering drugs and diet network; GWAS: Genome-wide association studies; POO: Parent-oforigin; QTDT: Quantitative transmission disequilibrium test; SNP: Single nucleotide polymorphism; TG: Triglyceride

\section{Acknowledgements}

We want to acknowledge Achilleas Pitsillides for his computing assistance.

\section{Funding}

Publication of the proceedings of Genetic Analysis Workshop 20 was supported by National Institutes of Health grant R01 GM031575.

\section{Availability of data and materials}

The data that support the findings of this study are available from the Genetic Analysis Workshop (GAW), but restrictions apply to the availability of these data, which were used under license for the current study. Qualified researchers may request these data directly from GAW.

\section{About this supplement}

This article has been published as part of BMC Genetics Volume 19 Supplement 1, 2018: Genetic Analysis Workshop 20: envisioning the future of statistical genetics by exploring methods for epigenetic and pharmacogenomic data. The full contents of the supplement are available online at https://bmcgenet. biomedcentral.com/articles/supplements/volume-19-supplement-1.

\section{Authors' contributions}

CS and JD conceived of the project. CS and SL performed statistical analyses. CS drafted the manuscript. All authors read, provided critical review of the manuscript, and approved the final manuscript.

Ethics approval and consent to participate

Not applicable.

\section{Consent for publication}

Not applicable.

\section{Competing interests}

The authors declare that they have no competing interests.

\section{Publisher's Note}

Springer Nature remains neutral with regard to jurisdictional claims in published maps and institutional affiliations.

Published: 17 September 2018

\section{References}

1. Ali O, Cerjak D, Kent JW Jr, James R, Blangero J, Carless MA, Zhang Y. Methylation of SOCS3 is inversely associated with metabolic syndrome in an epigenome-wide association study of obesity. Epigenetics. 2016;11(9):699-707.

2. Das M, Irvin MR, Sha J, Aslibekyan S, Hidalgo B, Perry RT, Zhi D, Tiwari HK, Absher D, Ordovas JM, et al. Lipid changes due to fenofibrate treatment are not associated with changes in DNA methylation patterns in the GOLDN study. Front Genet. 2015;6:304.

3. Das M, Sha J, Hidalgo B, Aslibekyan S, Do AN, Zhi D, Sun D, Zhang T, Li S, Chen W, et al. Association of DNA methylation at CPT1A locus with metabolic syndrome in the genetics of lipid lowering drugs and diet network (GOLDN) study. PLoS One. 2016;11(1):e0145789.

4. Irvin MR, Zhi D, Joehanes R, Mendelson M, Aslibekyan S, Claas SA, Thibeault KS, Patel N, Day K, Jones LW, et al. Epigenome-wide association study of fasting blood lipids in the genetics of lipid-lowering drugs and diet network study. Circulation. 2014;130(7):565-72.

5. Lai CQ, Wojczynski MK, Parnell LD, Hidalgo BA, Irvin MR, Aslibekyan S, Province MA, Absher DM, Arnett DK, Ordovás JM, et al. Epigenome-wide association study of triglyceride postprandial responses to a high-fat dietary challenge. J Lipid Res. 2016;57(12):2200-7.

6. Sayols-Baixeras S, Subirana I, Lluis-Ganella C, Civeira F, Roquer J, Do AN, Absher D, Cenarro A, Muñoz D, Soriano-Tárraga C, et al. Identification and validation of seven new loci showing differential DNA methylation related to serum lipid profile: an epigenome-wide approach. The REGICOR study. Hum Mol Genet. 2016;25(20):4556-65.

7. Predazzi IM, Sobota RS, Sanna S, Bush WS, Bartlett J, Lilley JS, Linton MF, Schlessinger D, Cucca F, Fazio S, et al. Sex-specific parental effects on offspring lipid levels. J Am Heart Assoc. 2015;4(7):e001951.

8. Sarnowski C, Laprise C, Malerba G, Moffatt MF, Dizier MH, Morin A, Vincent $\mathrm{QB}$, Rohde K, Esparza-Gordillo J, Margaritte-Jeannin P, et al. DNA methylation within melatonin receptor 1A (MTNR1A) mediates paternally transmitted genetic variant effect on asthma plus rhinitis. J Allergy Clin Immunol. 2016;138(3):748-53.

9. Abecasis GR, Cardon LR, Cookson WO. A general test of association for quantitative traits in nuclear families. Am J Hum Genet. 2000;66(1):279-92.

10. Whittaker JC, Gharani N, Hindmarsh P, McCarthy MI. Estimation and testing of parent-of-origin effects for quantitative traits. Am J Hum Genet. 2003; 72(4):1035-9.

11. Liu Y, Aryee MJ, Padyukov L, Fallin MD, Hesselberg E, Runarsson A, Reinius L, Acevedo N, Taub M, Ronninger M, et al. Epigenome-wide association data implicate DNA methylation as an intermediary of genetic risk in rheumatoid arthritis. Nat Biotechnol. 2013;31(2):142-7.

12. Kathiresan S, Melander O, Guiducci C, Surti A, Burtt NP, Rieder MJ, Cooper GM, Roos C, Voight BF, Havulinna AS, et al. Six new loci associated with blood low-density lipoprotein cholesterol, high-density lipoprotein cholesterol or triglycerides in humans. Nat Genet. 2008;40(2):189-97.

13. Teslovich TM, Musunuru K, Smith AV, Edmondson AC, Stylianou IM, Koseki M, Pirruccello JP, Ripatti S, Chasman DI, Willer CJ, et al. Biological, clinical and population relevance of 95 loci for blood lipids. Nature. 2010;466(7307):707-13. 